\title{
Ultra-Specific Enrichment of GST-Tagged Protein by GSH-Modified Nanoparticles
}

\author{
Yeon-Ji Lee, Jong-Moon Park, Jiyoung Huh, Minsik Kim, Je-Sun Lee, Arudra Palani, Kwangyeol Lee, ${ }^{\text {and Sang-Won Lee* }}$
}

\author{
Department of Chemistry, Korea University, Seoul 136-701, Korea \\ *E-mail: sw_lee@korea.ac.kr (S.W.L),kylee1@korea.ac.kr (K.Y.L) \\ Received February 23, 2010, Accepted March 30, 2010
}

\begin{abstract}
The selective isolation of specific proteins from complex protein mixtures by nanoparticles is reported. Glutathionemodified superparamagnetic nanoparticles were used to purify specific proteins fused with glutathione S-transferase via enzyme-substrate interactions. They demonstrated greatly improved selectivity and efficiency over micron sized capturing beads. The ultra-specific enrichment of target proteins was confirmed by both SDS-PAGE and LC/ MS/MS experiments.
\end{abstract}

Key Words: Proteomics, Glutathione, Superparamagnetic, Nanoparticle, Separation

\section{Introduction}

Studies of extremely complex samples by conventional bottom-up proteomics methods are limited by the dynamic range and speed of LC/MS/MS analysis. ${ }^{1-7}$ Even with the current state of the art proteomics analysis platform, only a small fraction of proteome samples observed in MS events are being analyzed (i.e. undersampling). Proteins in low concentration are often precluded from being identified due to this undersampling. Improving techniques that allow enrichment with high specificity and efficiency towards lesser abundant subsets of the proteome would be a significant advancement, with useful applications in, for example, finding patient-specific disease biomarkers from plasma. ${ }^{8-12}$

Enrichment techniques based on interactions of small molecules with enzymes might provide valuable information for drug discovery and personalized cancer therapy. ${ }^{13-21}$ Conventionally, target proteins have been captured by specific interactions on micron sized beads surface-modified by small molecules. ${ }^{22}$ For example, enzyme-tagged proteins such as GST (glutathione S-transferase)- and His (Histidine) can be specifically enriched by this bead method. ${ }^{23-27}$ Although the established micro-bead techniques are widely used, the separation of little abundant proteome samples with very high purity is difficult by this method because the captured proteome sample is largely contaminated by other non-specifically interacting proteins. This hinders detailed analysis.

A bead surface of micrometer curvature cannot provide suitable structural parameters for effective interaction between small molecules and enzymes' active sites. Furthermore, microbeads with roughened surfaces might have dimples or pores, greatly complicating the purification; more rigorous washing than is possible is required to remove completely the nonspecifically interacting proteins trapped within the surface pores of the microbeads. Nanoparticles, on the other hand, with very small diameters, can exhibit neither such surface pores nor the arising complications of structure-driven contamination. Also, the active sites used for specific binding in proteins are best recognized by nanoparticles, a promising property that spurs the development of nanoparticle-based proteome enrichments.
In particular, nanoparticles have been successfully demonstrated to isolate cysteinyl proteins by specific chemical reactionbased enrichment with minimal, if any, contamination from non-specifically interacting proteins. ${ }^{28,29}$ Thus, nanoparticles might be able to provide an ideal environment for the enrichment of proteins through specific enzyme-substrate interactions. In order to test this, GSH (glutathione)-modified magnetic $\mathrm{Fe}_{3} \mathrm{O}_{4} @$ $\mathrm{SiO}_{2}(\mathrm{ca} .30 \mathrm{~nm})$ nanoparticles were prepared for the enrichment of GST-tagged proteins. Thusly enriched protein sample was essentially devoid of any contamination, a result superior to those from conventional microbead techniques.

\section{Experimental}

Materials. GST-tagged ubiquitin (GST-Ub) was purchased from Calbiochem (La Jolla, CA, USA). Yeast enolase and glutathione were purchased from Sigma (St. Louis, MO, USA). Sequencing-grade modified trypsin was purchased from Promega (Madison, WI, USA). Oleic acid (90\%), Igepal CO-520, 1,2-dimethoxyethane (99.5\%), $\mathrm{N}$-[3-(trimethoxysilyl)propyl]ethylenediamine (80\%), 3-(trihydroxysilyl)propylmethyl phosphonate (42\%) were purchased from Aldrich. $N$-succinimidyln3(2-pyridyldithio)propionate (SPDP) and succinimidyl 6-(3-[2pyridldithio]-propionamido)hexanoate (LC-SPDP) were purchased from Calbiochem.

Preparation of glutathione-functionalized $\mathrm{Fe}_{3} \mathrm{O}_{4} @ \mathrm{SiO}_{2}$ nanoparticles (GSH-NPs). A previously reported synthesis of $\mathrm{Fe}_{3} \mathrm{O}_{4}$ (a) $\mathrm{SiO}_{2}$ nanoparticles, which were surface functionalized by $\mathrm{N}$-succinimidyl 3-(2-pyridyldithio)propionate (SPDP) for specific enrichment of free-thiol containing peptides, was used. ${ }^{28}$ Briefly, the $29.6( \pm 2.1)-\mathrm{nm} \mathrm{Fe}_{3} \mathrm{O}_{4} @ \mathrm{SiO}_{2} \mathrm{NPs}$ were surface modified with 3-(trihydroxysliyl)propylmethylphosphonate (THPMP) and $N$-[3-(trimethoxysilyl)propyl]-ethylenediamine (AEAPTMS). These $\mathrm{Fe}_{3} \mathrm{O}_{4} @ \mathrm{SiO}_{2}$ NPs coated with AEAPTMS and THPMP $(100 \mathrm{mg})$ were dispersed in methanol $(9.5 \mathrm{~mL})$ and sonicated for $1 \mathrm{~h}$. A solution of either $N$-succinimidyl 3(2-pyridyldithio) propionate $(0.2 \mu \mathrm{mol}$ for $1 \mathrm{mg}$ of NPs; short chain (SC) SPDP ,Calbiochem) or succinimidyl 6-(3-[2-pyridyldithio]-propionamido)hexanoate $(0.2 \mu \mathrm{mol}$ for $1 \mathrm{mg}$ of NPs; long chain (LC) SPDP, Calbiochem) dissolved in DMSO 
$(500 \mu \mathrm{L})$ was added to the dispersed $\mathrm{Fe}_{3} \mathrm{O}_{4} @ \mathrm{SiO}_{2}$ NPs coated with AEAPTMS and THPMP and the resulting solution was stirred mechanically ( $350 \mathrm{rpm})$ for $48 \mathrm{~h}$ at room temperature. The SC-SPDP-NPs and LC-SPDP-NPs were washed with $\mathrm{MeOH}(3 \times 25 \mathrm{~mL})$ and dried before they were reacted with glutathione. The $50 \mathrm{mM}$ glutathione solution was prepared by dissolving dried glutathione in $50 \mathrm{mM}$ Tris and $10 \mathrm{mM}$ EDTA (Tris-HCl buffer; $\mathrm{pH}$ 7.5). After dissolving glutathione in Tris$\mathrm{HCl}$, the $\mathrm{pH}$ of the solution was adjusted to 7.5 by $100 \mathrm{mM}$ $\mathrm{NaOH}$. The glutathione solutions were blended with SC-SPDPNPs or LC-SPDP-NPs ( $5 \mathrm{mg}$ ) in Tris-HCl buffer ( $\mathrm{pH} 7.5,200$ $\mu \mathrm{L}$ ) for $2 \mathrm{hr}$ at $900 \mathrm{rpm}$ at room temperature. The supernatant was removed and the remaining NPs were washed with Tris$\mathrm{HCl}$ buffer $(200 \mu \mathrm{L})$. Any remaining free glutathione was removed from the NPs' surfaces by two steps of washings each with $1 \%$ acetic acid $(200 \mu \mathrm{L}), 1.5 \mathrm{M} \mathrm{NaCl}(200 \mu \mathrm{L}), 80 \%$ acetonitrile $(200 \mu \mathrm{L})$, and Tris- $\mathrm{HCl}(200 \mu \mathrm{L})$. The glutathione functionalized NPs (SC-GSH-NPs or LC-GSH-NPs, Figure 1) were stored in Tris- $\mathrm{HCl}$ buffer to give a final concentration of particles of $0.5 \mathrm{mg} / 20 \mu \mathrm{L}$ at $4{ }^{\circ} \mathrm{C}$.

Purification of GST tagged protein from protein mixtures. A two-protein mixture was prepared by mixing $5 \mu \mathrm{g}$ of GST$\mathrm{Ub}$ with $5 \mu \mathrm{g}$ of yeast enolase protein $(5 \mu \mathrm{g})$ in Tris-HCl buffer. In order to mimic complex protein mixtures, yeast proteome mixture was produced by mixing the soluble fraction of yeast proteome $^{30}$ and GST-Ub $(3 \mu \mathrm{g})$; human serum mixture was similarly produced by mixing a depleted human serum and GST-Ub $(3 \mu \mathrm{g})$. The depleted serum sample was prepared by using the multiple affinity removal (MARS, Agilent Technologies, Santa Clara, CA) column on a crude serum sample. The crude serum sample was first diluted 5 times with Buffer A and loaded onto the MARS column at a flow rate of 0.5 $\mathrm{mL} / \mathrm{min}$. After washing the column with Buffer A (10 min), the bound proteins were eluted with Buffer B (5 min). The resultant proteins were incubated with trypsin $(50: 1)$ at $37{ }^{\circ} \mathrm{C}$ overnight and $0.1 \%$ formic acid was added. The $20 \mu \mathrm{L}$ of glutathione-functionalized $\mathrm{Fe}_{3} \mathrm{O}_{4} @ \mathrm{SiO}_{2}$ nanoparticle (GSHNPs) dispersion $(0.5 \mathrm{mg} / 20 \mu \mathrm{L}$ in Tris- $\mathrm{HCl}$ buffer $)$ was taken from the stock particle dispersion. Each protein sample was mixed with glutathione functionalized $\mathrm{Fe}_{3} \mathrm{O}_{4} @ \mathrm{SiO}_{2}$ nanoparticles (GSH-NPs) and then incubated for $10 \mathrm{~min}$ in thermomixer (Eppendorf) with gentle mixing at $4{ }^{\circ} \mathrm{C}$. Attachment of a small $\mathrm{SmCo}_{5}$ magnet to the outside of the sample tube allowed the dispersed GSH-NPs to be collected within a few seconds. After removing the supernatant, the remaining GSH-NPs were washed with $20 \mu \mathrm{L}$ of $40 \mathrm{mM}$ HEPES, $0.5 \%$ Triton X-100, $120 \mathrm{mM}$ $\mathrm{NaCl}, 1 \mathrm{mM}$ PMSF (Lysis buffer) by vortexing and sonicating for $10 \mathrm{sec}$ to remove contamination by non-specific interacting proteins. This washing was repeated twice. Further washing, with $500 \mathrm{mM}$ ammonium acetate solution, removed nonspecifically interacted proteins with phosphonate groups which give negative charge to the surfaces of the nanoparticles. The supernatants and captured proteins were digested with trypsin (promega) in Tris- $\mathrm{HCl}(\mathrm{pH} 7.5)$ and resultant tryptic peptides were diluted to a final concentration of $10 \mathrm{ng} / \mu \mathrm{L}$ in a solvent mixture of $2 \%$ acetonitrile, $0.2 \%$ TFA in water. A $1 \mu \mathrm{L}$ portion of the peptide solution was injected into a capillary RPLC/MS/ MS system for analysis. For SDS-PAGE gel electrophoresis analysis, the nano-captured protein was eluted from the GSHNPs with $20 \mu \mathrm{L}$ of $50 \mathrm{mM}$ free glutathione solution dissolved in Tris- $\mathrm{HCl}(\mathrm{pH} 7.5)$ and incubated for $4 \mathrm{hr}$ at $4{ }^{\circ} \mathrm{C}$ with gentle mixing. The GSH-NPs were then removed using a small SmCo5 magnet.

Purification of GST tagging protein from complex protein mixtures using glutathione sepharose $4 \mathrm{~B}$ beads having same function as glutathione NPs. The same protein mixtures as above, but with different degrees of complexity, were prepared by mixing $N$-terminal GST tagging ubiquitin and yeast enolase, $N$-terminal GST tagging ubiquitin and whole yeast proteome, and $N$-terminal GST tagging ubiquitin and whole human serum proteome. Glutathione sepharose 4B beads were washed and equilibrated for 10 min with PBS buffer in the spin columns (Pierce). Each protein mixture sample was incubated with prepared glutathione sepharose $4 \mathrm{~B}$ beads for $10 \mathrm{~min}$ at $4{ }^{\circ} \mathrm{C}$ in a thermomixer (Eppendorf) in the spin columns. After the separation of supernatant from the beads with low speed centrifugation, the beads were washed with $20 \mu \mathrm{L}$ of $40 \mathrm{mM}$ HEPES, $0.5 \%$ Triton X-100, $120 \mathrm{mM} \mathrm{NaCl}$ and $1 \mathrm{mM}$ PMSF (Lysis buffer) by vortexing for $10 \mathrm{sec}$ to remove non-specifically bound proteins. The two supernatants were combined for analysis. Captured proteins and supernatant were trypsinized for capillary RPLC/MS/MS analysis and eluted from the beads for SDSPAGE gel electrophoresis analysis by the above mentioned methods for the glutathione NPs.

Capillary RPLC/MS/MS experiments. The tryptic peptides of the GST tagging ubiquitin, resultant supernatants and purified proteins were analyzed using LTQ-FT (Thermo) and nano AUQUITY UPLC (Waters) systems. The nano AUQUITY UPLC system was equipped with an in-house capillary column $(75 \mu \mathrm{m}$ ID $\times 360 \mu \mathrm{m}$ OD $\times 75 \mathrm{~cm})$ packed with C18-bonded particles ( $3 \mu \mathrm{m}$ diameter, $300 \AA$ Å pore size, Phenomenex). Each sample of tryptic peptides was loaded onto the online SPE column for $6 \mathrm{~min}$ in solvent $\mathrm{A}(0.1 \%$ formic acid in water $)$ and eluted from the column with a 60 -min gradient of $10-50 \%$ solvent $\mathrm{B}(0.1 \%$ formic acid in acetonitrile) at a flow rate of $0.35 \mu \mathrm{L} / \mathrm{min}$. The LTQ-FT mass spectrometer was operated in a data-dependent mode in which one full MS (from $m / z 450$ to 1800 ) scan was followed by three MS/MS scans. The peptides were dissociated under the normalized collision energy of $35 \%$.

Proteomics data analysis. The LC/MS/MS data were subjected to a SEQUEST database search (Bioworks 3.2, Thermo) against yeast protein database (Saccharomyces cerevisiae, ftp:// genome-ftp.stanford.edu/pub/yeast/data_download/sequenc e/genomic_sequence/orf_protein/) or human international protein index database (IPI, v. 3.51, ftp://ftp.ebi.ac.uk). Before performing the database search with the SEQUEST search algorithm, the MS/MS data were filtered and refined through the algorithm of post-experiment monoisotopic mass filtering and refinement (PE-MMR). ${ }^{30}$ The peptide mass tolerance was $0.1 \mathrm{Da}$; the fragment ion mass tolerance was $\pm 1 \mathrm{Da}$. The modification parameters were set to +15.99492 for methionine oxidation.

The results of database searches were validated through the trans proteomic pipeline (TPP, ISB, http;//localhost/tpp-bin/ tpp_gui.pl). Peptide lists with false positive rates of $1 \%$ were used for the subsequent analyses. The masses and LC retention 
times of validated peptide IDs were searched against the unique mass class list (UMC list) ${ }^{30}$ in order to find a match within mass tolerance of $20 \mathrm{ppm}$ and retention time tolerance of 30 sec. The sum intensity, which is the summation of intensities of every corresponding peptide feature, of the matched UMC was assigned to the peptide ID as its observed intensity.

SDS-PAGE gel electrophoresis. SDS-PAGE was performed according to Laemmli's ${ }^{31}$ method with $12 \%$ acrylamide for the running gel and 5\% for the stacking gel in the presence of $0.1 \%$ SDS, without 2-mercaptoethanol. The SDS-PAGE gel was first run at a constant $60 \mathrm{~V}$ for $30 \mathrm{~min}$ and then $120 \mathrm{~V}$ for $1 \mathrm{hr}$ (Mini-PROTEANR Tetra Cell, Bio-Rad). Silver staining was done by the manufacture's recommended method. Briefly, proteins in the gel were fixed with $50 \% \mathrm{v} / \mathrm{v}$ methanol, $12 \%$ $\mathrm{w} / \mathrm{v}$ acetic acid, and $0.05 \% \mathrm{w} / \mathrm{v}$ formaldehyde overnight and washed three times with $50 \% \mathrm{v} / \mathrm{v}$ methyl alcohol for $20 \mathrm{~min}$. The sensitizing solution $(0.02 \% \mathrm{w} / \mathrm{v}$ sodium thiosulfate $)$ was applied for $1 \mathrm{~min}$ to increase sensitivity, and washed three times with distilled water. Proteins were stained with $0.1 \%$ silver nitrate staining solution for 20 - $30 \mathrm{~min}$, developed with $6 \% \mathrm{w} / \mathrm{v}$ sodium carbonate, $0.05 \% \mathrm{v} / \mathrm{v}$ formaldehyde to visualize the proteins. The reaction was stopped by applying a stopping solution of $50 \%$ methanol, $12 \% \mathrm{w} / \mathrm{v}$ acetic acid.

\section{Results and Discussion}

We synthesized two GSH-NPs with different spacer lengths (16.9 $\AA$ (SC-GSH-NP) and $24.9 \AA$ (LC-GSH-NP)) which are shown in Figure 1. Propylmethylphosphonate groups, added to provide sufficient water dispersity of the nanoparticles, ${ }^{32}$ are depicted as red dotted circles. Negligible difference was found in the capturing efficiencies of SC-GSH-NPs and LCGSH-NPs (Data not shown), indicating that the severe curvature of the nanoparticles provides sufficient space between surfacefixed GSH molecules to accommodate the large GST-tagged

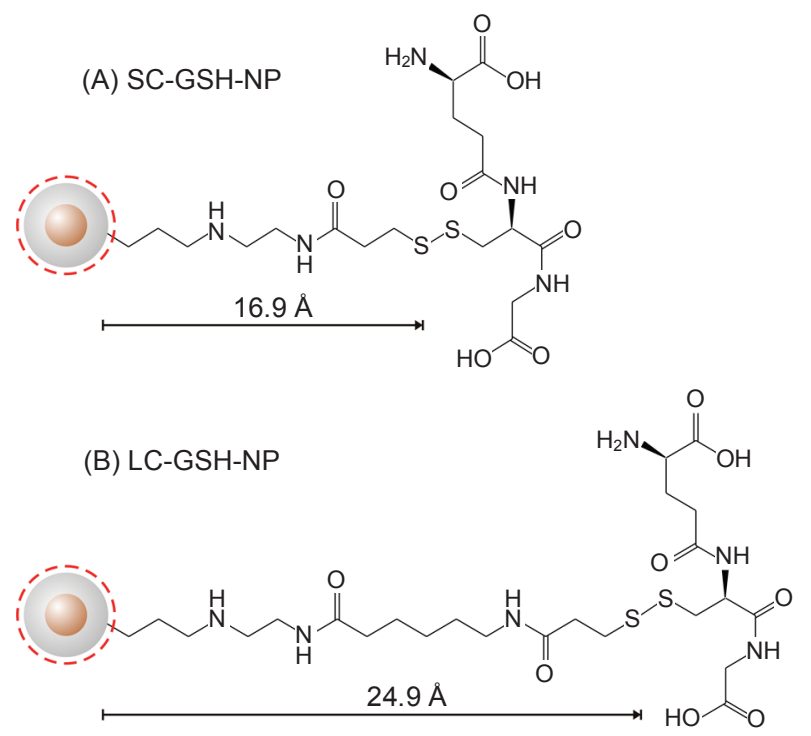

Figure 1. Structures of short chain GSH-NP (SC-GSH-NP) and long chain GSH-NP (LC-GSH-NP). The red dotted circle represents propylmethylphosphonate groups protein without requiring elongated spacers between capturing nanoparticles and the affixed small molecules.

Both SC- and LC-GSH-NPs demonstrated excellent capturing efficiency toward GST-tagged proteins. When the GSHNPs were added to GST-Ub solution, the supernatant showed no trace of GST-Ub. Also, elution from GSH-NPs recovered GST-Ubs close to the initial amount, as confirmed by SDSPAGE and LC/MS/MS experiments (Supplementary Figure 1, 2 and 3).

SC-GSH-NPs were applied to a two protein mixture of GSTtagged ubiquitin(GST-Ub) and yeast enolase. Figure 2 shows the SDS-PAGE (Figure 2A) and LC/MS/MS data of the supernatant (Figure 2B, lane 2) and the captured sample (Figure 2B, lane 3).

After mixing the protein mixtures with GSH-NPs and their subsequent magnetic separation, the supernatants displayed no trace of GST-Ub in the resulting gel lane. A series of washings removed any non-specifically interacting proteins, allowing the captured proteins to be released from the NPs by the addition of free glutathione solution and incubation for $4 \mathrm{hr}$ at $4{ }^{\circ} \mathrm{C}$ with gently mixing. The resulting sample showed a single spot corresponding to the GST-Ub (Figure 2A, lane 3). LC/MS/ MS experiments were performed on both lane 2 and lane 3 samples after each of them was superlatively subjected to tryptic digestion. When compared with data obtained from analyzing pure tryptic GST-Ub peptides, the resultant LC/MS/MS chromatogram obtained from the lane 3 sample showed no contaminant peaks from non-specific tryptic enolase peptides while the supernatant (lane 2) showed only evidence of enolase peptides, suggesting that the GSH-NPs acted excellently in targeting and capturing GST-tagged proteins from a two protein mixture.

GSH-NPs were also used to capture GST-tagged protein from higly complex proteome samples. First, GSH-NPs were used to purify GST-Ub from the whole yeast proteome. In order to compare the selectivity of GSH-NPs with prior art micron size particles, the same capturing experiments were performed with glutathione sepharose 4B beads. Figure 3 shows SDS/PAGE and LC/MS/MS analyses after capturing experiments using glutathione sepharose 4B beads (lanes 2 and 3 ) and GSH-NPs (lanes 4 and 5).

While micron size beads resulted in several non-GST-Ub bands on the gel lane of the captured sample, those of GSH-NPs show no traces of other protein spots, indicating higher capturing selectivity of GSH-NPs over conventional micron size beads. The samples captured by GSH-NPs gave markedly different LC/MS/MS chromatograms from micron size beads (Figure 3B) suggesting that the two particles captured significantly different proteins. Contrary to the SDS/PAGE data, where GSH-NPs had no trace of capturing non-specific proteins, both the LC/MS/ MS data showed evidence of GST-Ub, the target protein, along with other proteins. In order to estimate the capturing efficiency and selectivity of both methods, the sum intensities of the validated peptides of each identified protein were calculated and normalized with those observed from pure standard GST$\mathrm{Ub}$. Figure $3 \mathrm{C}$ compares the relative sum intensities of the proteins identified from both methods. While the LC/MS/MS data from GSH-NPs showed sum intensities of peptides from 
(A)

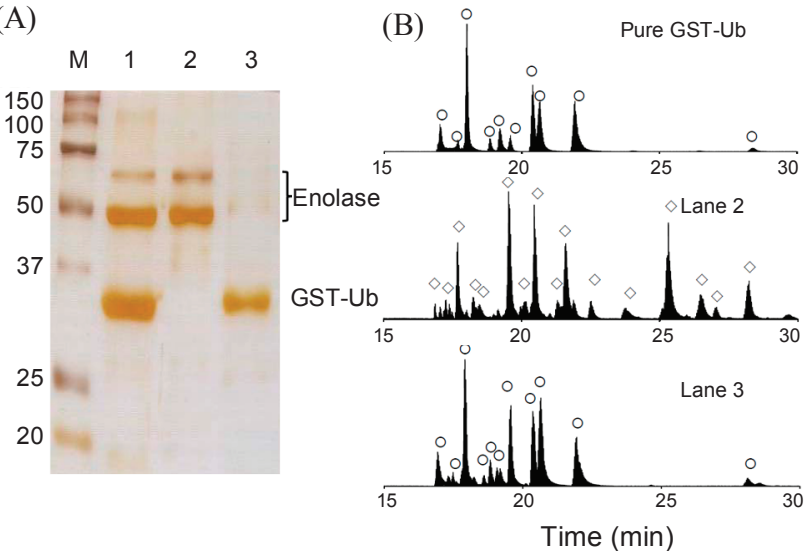

Figure 2. SDS/PAGE and LC/MS/MS analyses of capturing GSTtagged protein (GST-Ub, $35 \mathrm{kD}$ ) from a simple two protein mixture. A. SDS-PAGE data: Molecular weight marker (lane M), two protein mixture (lane 1), supernatants after mixing with SC-GSH-NP (lane 2) and after capturing and eluting from SC-GSH-NP (lane 3). B. Base peak chromatograms of LC/MS/MS experiments of pure tryptic GST$\mathrm{Ub}$ and tryptic peptides from lane 2 and lane 3 samples. The tryptic peptides of GST-Ub and enolase are denoted by the symbols $\bigcirc$ and $\diamond$, respectively. For clarity, not all the peptides have been labeled.
(A)

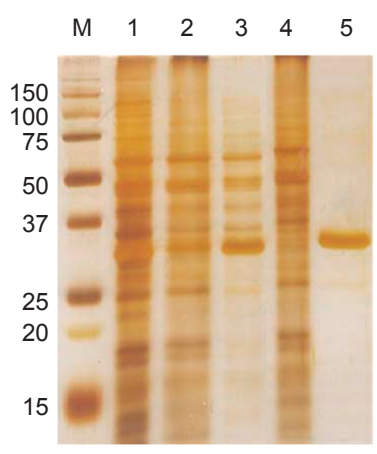

(B)

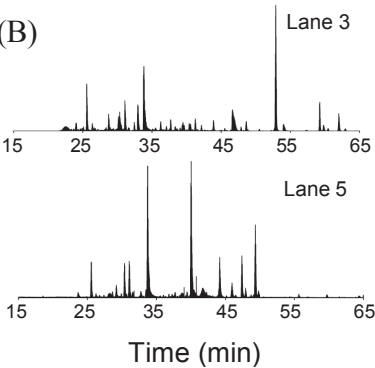

(C)

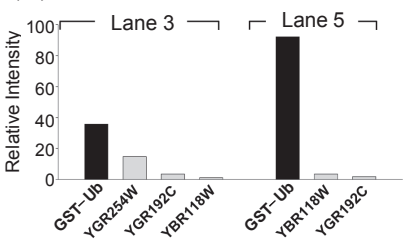

Figure 3. SDS/PAGE and LC/MS/MS analyses of capturing GST -Ub from a protein mixture of GST-Ub and yeast proteome. A. SDSPAGE data: Molecular weight marker (lane M), yeast proteome and GST-Ub mixture (lane 1), supernatants after mixing with glutathione sepharose $4 \mathrm{~B}$ beads (lane 2), and capturing and eluting from glutathione sepharose 4B beads (lane 3), supernatants after mixing with SC-GSHNP (lane 4), and after capturing and eluting from SC-GSH-NP (lane 5). B. Base peak chromatograms of LC/MS/MS experiments of tryptic peptides from lane 3 and lane 5 samples. C. Relative sum intensities of peptides of proteins identified by LC/MS/MS experiments on lane 3 and lane 5 samples. For clarity, only proteins of $1 \%$ or higher of pure GST-Ub intensity were only shown.

GST-Ub were dominant in the presence of minor nonspecific proteins, those from micron size beads identified an abundance of many other yeast proteins. The observed sum intensities after capturing with GSH-NPs are very close to those of pure

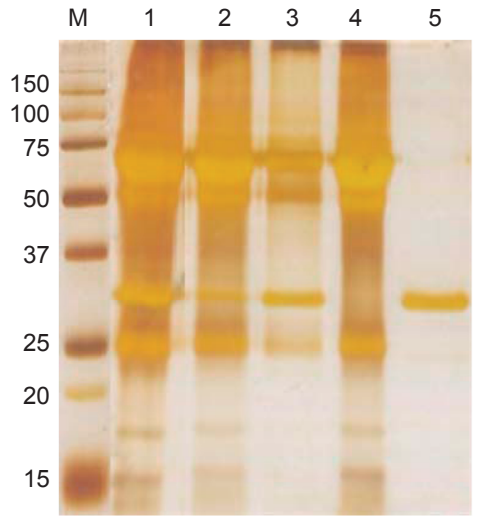

Figure 4. SDS/PAGE analyses of capturing GST-Ub from a mixture of GST-Ub and the human serum proteome. A. SDS-PAGE data. Molecular weight marker (lane M), serum proteome with GST-Ub (lane 1), supernatant after mixing with glutathione sepharose 4B beads (lane 2), and after capturing and eluting from glutathione sepharose $4 \mathrm{~B}$ beads (lane 3), supernatant after mixing with SC-GSH-NP (lane 4) and after capturing and eluting from SC-GSH-NP (lane 5).

GST-Ub, indicating that the GSH-NPs nearly completely captured the GST-Ub.

GSH-NPs were used to enrich GST-Ub from a depleted human serum. Figure 4 displays the resultant SDS/PAGE data of the captured GST-Ub. Despite the extreme complexity of the serum proteome, GSH-NPs efficiently captured the target protein with exceptional selectivity. LC/MS/MS experiments showed the GSH-NPs predominantly captured peptides from the target protein (Supplementary Figures 4 and 5).

\section{Conclusion}

Glutathione-functionalized superparamagnetic nanoparticles (GSH-NPs) were synthesized and used to capture and purify GST-tagged proteins from complex protein mixtures. They exhibited greatly improved selectivity and efficiency over prior art micron size capturing beads. Such exceptionally selective and efficient protein capturing should enhance biological confirmation of captured proteins, not only by allowing the capture of lesser abundant candidate proteins but also increasing the concentration of captured proteins.

Acknowledgments. This work was supported by grants from the 21C Frontier for Functional Proteomics (FPR08A1-010), Converging Research Center for Mass Spectrometric Diagnosis, the Fundamental R\&D Program for Core Technology of Materials funded by the Ministry of Knowledge Economy to SWL and MOST (KOSEF M10755020001-08N5502-00110), KRF2008-314-C00234, MIHWAF (the Korea Health 21 R\&D Project: A085136) to KL.

Supporting Information Available. Additional protein separation results by using GSH-NPs are available on request from the correspondence author. 


\section{References}

1. Tabb, D. L.; MacCoss, M. J.; Wu, C. C.; Anderson, S. D.; Yates, J. R. 3rd. Anal. Chem. 2003, 75(10), 2470-2477.

2. Higgs, R. E.; Knierman, M. D.; Gelfanova, V.; Butler, J. P.; Hale, J. E. J. Proteome. Res. 2005, 4(4), 1442-1450.

3. Wang, G.; Wu, W. W.; Zeng, W.; Chou, C. L.; Shen, R. F. J. Proteome. Res. 2006, 5(5), 1214-1223.

4. Cutillas, P. R.; Vanhaesebroeck, B. Mol. Cell. Proteomics 2007, 6(9), 1560-1573

5. Old, W. M.; Meyer-Arendt, K.; Aveline-Wolf, L.; Pierce, K. G.; Mendoza, A.; Sevinsky, J. R.; Resing, K. A.; Ahn, N. G. Mol. Cell. Proteomics 2005, 4(10), 1487-1502.

6. Mueller, L. N.; Brusniak, M. Y.; Mani, D. R.; Aebersold, R. J. Proteome. Res. 2008, 7(1), 51-61.

7. Qian, W. J.; Jacobs, J. M.; Liu, T.; Camp, D. G., 2nd.; Smith, R. D. Mol. Cell. Proteomics 2006, 5(10), 1727-1744.

8. Jalili, P. R.; Ball, H. L. J. Am. Soc. Mass. Spectrom. 2008, 19(5), 741-750

9. Zhao, L.; Wu, R.; Han, G.; Zhou, H.; Ren, L.; Tian, R.; Zou, H. J. Am. Soc. Mass. Spectrom. 2008, 19(8), 1176-1186.

10. Reid, G. E.; Roberts, K. D.; Simpson, R. J.; O'Hair, R. A. J. Am. Soc. Mass. Spectrom. 2005, 16(7), 1131-1150.

11. Whiteaker, J. R.; Zhao, L.; Zhang, H. Y.; Feng, L. C.; Piening, B. D.; Anderson, L.; Paulovich, A. G. Anal. Biochem. 2007, 362(1), 44-54.

12. Hsieh, H. C.; Sheu, C.; Shi, F. K.; Li, D. T. J. Chromatogr. A 2007, 1165(1-2), 128-135.

13. Gao, J.; Gu, H.; Xu, B. Acc. Chem. Res. 2009, 42(8), 1097-1107.

14. Gao, J.; Liang, G.; Cheung, J. S.; Pan, Y.; Kuang, Y.; Zhao, F.; Zhang, B.; Zhang, X.; Wu, E. X.; Xu, B. J. Am. Chem. Soc. 2008, 130(35), 11828-11833.

15. Yang, J.; Lee, J.; Kang, J.; Oh, S. J.; Ko, H. J.; Son, J. H.; Lee, K.; Suh, J. S.; Huh, Y. M.; Haam, S. Adv. Mater. 2009, 21, 1-4.

16. Yang, J.; Lee, C. H.; Ko, H. J.; Suh, J. S.; Yoon, H. G.; Lee, K.;
Huh, Y. M.; Haam, S. Angew. Chem. Int. Ed. Engl. 2007, 46(46), 8836-8839.

17. Ferrari, M. Nat. Rev. Cancer 2005, 5(3), 161-171.

18. Michalet, X.; Pinaud, F. F.; Bentolila, L. A.; Tsay, J. M.; Doose, S.; Li, J. J.; Sundaresan, G.; Wu, A. M.; Gambhir, S. S.; Weiss, S. Science 2005, 307(5709), 538-444.

19. Lee, J. H.; Lee, K.; Moon, S. H.; Lee, Y.; Park, T. G.; Cheon, J. Angew. Chem. Int. Ed. Engl. 2009, 48(23), 4174-4179.

20. Lee, H.; Yu, M. K.; Park, S.; Moon, S.; Min, J. J.; Jeong, Y. Y.; Kang, H. W.; Jon, S. J. Am. Chem. Soc. 2007, 129(42), 1273912745.

21. MacDiarmid, J. A.; Amaro-Mugridge, N. B.; Madrid-Weiss, J.; Sedliarou, I.; Wetzel, S.; Kochar, K.; Brahmbhatt, V. N.; Phillips, L.; Pattison, S. T.; Petti, C.; Stillman, B.; Graham, R. M.; Brahmbhatt, H. Nat. Biotechnol. 2009, 27(7), 643-651.

22. Ford, C. F.; Suominen, I.; Glatz, C. E. Protein Expr. Purif. 1991, 2(2-3), 95-107.

23. Rodal, A. A.; Duncan, M.; Drubin, D. Methods Enzymol. 2002, $351,168-172$

24. Hengen, P. N. Trends Biochem. Sci. 1996, 21(10), 400-401.

25. Murray, A. M.; Kelly, C. D.; Nussey, S. S.; Johnstone, A. P. J. Immunol. Methods 1998, 218(1-2), 133-139.

26. Jalili, P. R.; Sharma, D.; Ball, H. L. J. Am. Soc. Mass. Spectrom. 2007, 18(6), 1007-1017.

27. Ross, A. R. Methods Enzymol. 2007, 423, 549-572.

28. Palani, A.; Lee, J. S.; Huh, J.; Kim, M.; Lee, Y. J.; Chang, J. H.; Lee, K.; Lee, S. W. J. Proteome. Res. 2008, 7(8), 3591-3596.

29. Lee, I. S.; Lee, N.; Park, J.; Kim, B. H.; Yi, Y. W.; Kim, T.; Kim, T. K.; Lee, I. H.; Paik, S. R.; Hyeon, T. J. Am. Chem. Soc. 2006, 128(33), 10658-10659.

30. Shin, B.; Jung, H. J.; Hyung, S. W.; Kim, H.; Lee, D.; Lee, C.; Yu, M. H.; Lee, S. W. Mol. Cell. Proteomics 2008, 7(6), 1124-1134.

31. Laemmli, U. K. Nature 1970, 227(5259), 680-685.

32. Bagwe, R. P.; Hilliard, L. R.; Tan, W. Langmuir 2006, 22(9), 43574362. 